\title{
Informed Matter, Design and its Relationship to Force Dynamics
}

\section{SIGRADI2018 TECHNOPOLITICAS \\ xxii congresso da sociedade iberoamericana de gráfica digital 22th conference of the iberoamerican society of digital graphics 07|08|09|novembro|2018 iau usp | são carlos | sp br}

\author{
PhD(c)Arch Juan Manuel Villa Carrero \\ Universidad Francisco de Paula Santander - UFPS | Colombia | juanmanuelvc@ufps.edu.co \\ dlab@ufps.edu.co
}

MArch Álvaro Maldonado Montagut

Universidad Francisco de Paula Santander - UFPS | Colombia |

alvaroenriquemm@ufps.edu.co

\begin{abstract}
The form can be described as the action of a force on matter, so this research turned to the essence of this fact of reality to objectively confront it, explore its methods of representation and challenge our design responses. The spaces of topological order that were perceived in the results move away from concrete imaginaries, symbols and metaphors; the design was the result of the information and the matter acted as an informed mass that was modeled from different forces that acted on it. This general research had as its general objective to understand the real, this reality understood as an abstract formal structure, which is based on complex scientific and experimental work, within the digital world.
\end{abstract}

Keywords: Forces Dynamics, Simulation, Design, Technologies, Data.

\section{INTRODUCCIÓN}

La correlación entre la materia y su resistencia acomoda sus espacios, derivando su forma, la cual, a su vez, es el resultado de las dinámicas de fuerzas. Este conocimiento de la "estructura de las estructuras" ampliamente tratado en el siglo XX por ingenieros como R. Le Ricolai (1973), R. B. Fuller (1975) o biólogos como D'arcy Thompson (1942), permite la optimización, y el empleo mínimo de la materia estructural y su hipotético campo de aplicaciones escalares en el diseño.

Sin embargo, la dislocación entre una "realidad objetiva" (cercana al mundo científico) y la realidad experiencial (cercano a lo subjetivo y sensorial) en las prácticas comunes de diseño afectan la comprensión de estas disposiciones de la materia, y por ende, el entendimiento de la geometría de sus vacíos, o de un espacio continuo y conectivo (topológico) que admita respuestas de diseño más eficientes.

Así pues, planteamos la siguiente problemática: ¿debemos objetivar la materia para obtener respuestas de diseño más eficiente en relación con la dinámica de fuerzas?"

Slavoj Zizeck, propone que, "Debemos desarrollar, creo, un nuevo materialismo abstracto más terrorífico, una especie de universo matemático donde no hay nada. Solo hay fórmulas, formularios técnicos, etc". (Zizek, 2014)
Por tanto, el objeto de esta investigación es explicar el trabajo de informar la materia en el mundo digital en relación con las dinámicas de fuerzas y su sustancia, las cuales, configuran su forma, para así, dar respuesta a nuestro problema inicial. En particular, esta labor se explica en este texto a través de la aplicación de medios tecnológicos digitales, que operan como bienes comunes, que potencian la práctica creativa de "espacios imaginados a los construidos", dentro de un "universo matemático", un modo nuevo de hacer que se contrapone a las técnicas dominantes de representación.
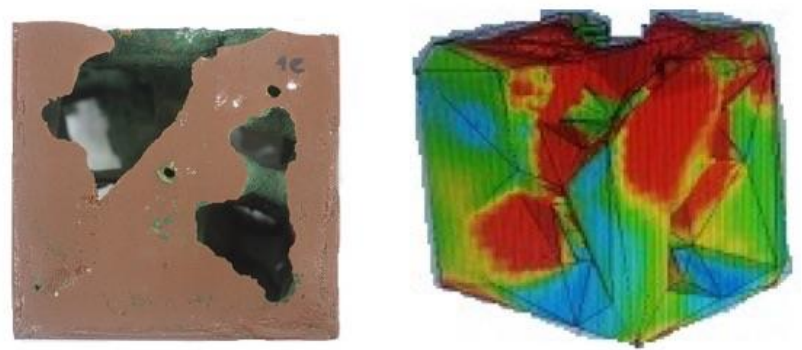

Figura 1: Probeta de material compuesto (probeta $10 \mathrm{~cm} \times 10 \mathrm{~cm}$ $10 \mathrm{~cm})$ y simulación de la probeta en un entorno digital (Rhinoceros - Plugin Scan\&Slove) para el desarrollo de un objeto proyectual arquitectónico. Fuente: autores.

Por ejemplo, de manera específica, inicialmente este trabajo pretende la codificación de la materia y la generación de un proto-modelo digital que explique sus geometrías. En segundo lugar, esta investigación busca obtener resultados numéricos del comportamiento mecánico del material desarrollado, exponiéndolo a 
fuerzas de compresión que ponga a prueba la correlación entre la materia y su resistencia. Y por último, pretende exponer la materia informada a la realidad abstracta de la simulación, o a una hipótesis de la realidad que permita el escalamiento de sus resultados.

\section{METODOLOGÍA}

Para comprender mejor el desarrollo metodológico la investigación plantea una etapa preliminar, que corresponde a la elaboración de un material compuesto (probeta $10 \mathrm{~cm} \times 10 \mathrm{~cm} 10 \mathrm{~cm}$ ) mediante método científico, para el desarrollo del objeto proyectual planteado en la asignatura del taller de diseño (2do año universitario de la carrera de arquitectura). (Figura 1).

Es importante destacar que la producción material está atravesada por dos intensiones propias del estado de la materia y el espacio, las cuales perfilan las intenciones en esta fabricación y son las siguientes: a. Incorporar dentro del volumen de material, una zona de vacío en continuidad permanente, otorgándole cualidades de orden topológico, b. A partir del estudio de los elementos elegidos para esta nueva conformación, definir las intenciones mecánicas, con las que se desea abordar el nuevo compuesto material.

Hecha esta salvedad a continuación, describimos las fases fundamentales del método:

\section{FASE 1}

Proceso Digitalización - Modelo: El proceso de digitalización que se realiza sobre la muestra material creada de forma análoga (Probeta), busca la mayor fidelidad posible en la información morfológica y permite la múltiples simulaciones y manipulaciones posteriores, en el entorno informático, con la posibilidad de volver al modelo inicial y resguardar sus atributos.

Para este proceso se escanea la muestra física material, mediante el uso del escáner 3D, un EinScan-Pro, marca Shining y cámara para captura de colores y texturas, instrumento tecnológico que se encuentran dentro del laboratorio SIMU-lab, de la Universidad Francisco de Paula Santander. (Figura 2)

\section{FASE 2}

Proceso Análogo - Data: Esta fase nos permite obtener resultados numéricos del comportamiento mecánico del material compuesto desarrollado; información numérica, fundamental para definir los comportamientos de resistencia del material creado en relación a los elementos materiales que la componen.

Dentro de los resultados que se obtienen en este ensayo de compresión, podemos encontrar el modo elástico expresado en Mpa, el cual, es la zona de deformación en $\mathrm{mm} / \mathrm{mm}$, que, logra el material antes de su límite de rotura. El límite de rotura se define como la máxima tensión ( $\mathrm{N}$ ) alcanzada del material, el cual, se encuentra en el punto más alto de la curvatura $(T)$ de la gráfica de los análisis de resistencia mecánica.

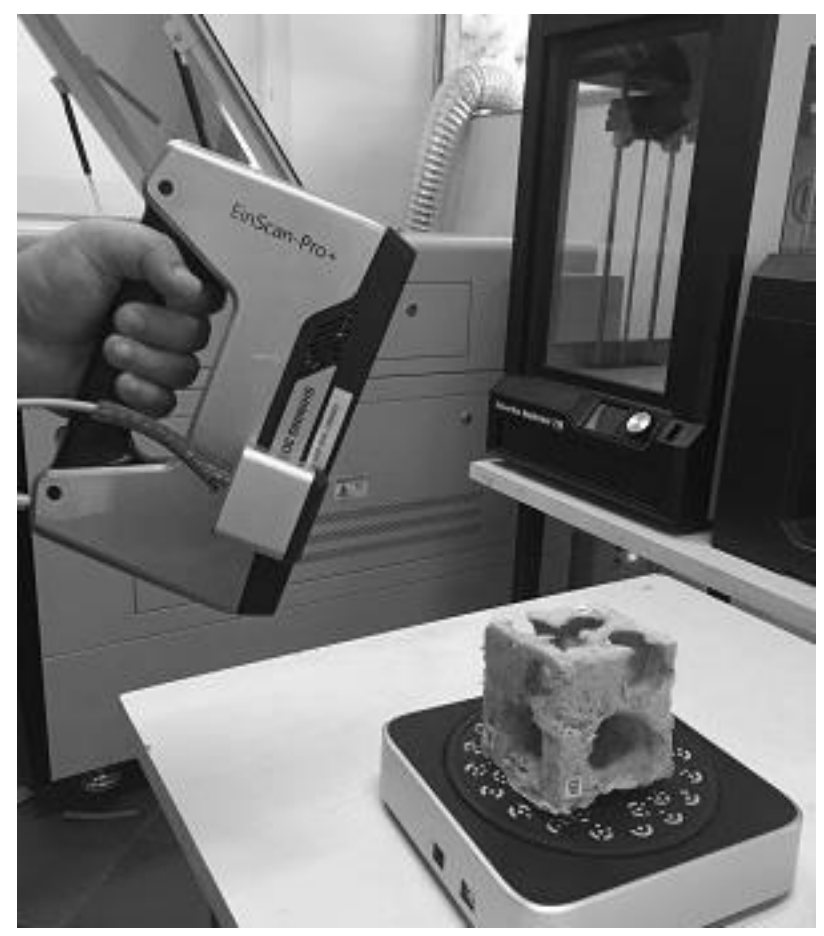

Figura 2: Laboratorio de investigación en diseño - UFPS, proceso de escaneo 3D de las probetas de material compuesto y su vacío interior en continuidad permanente. Fuente: autores.

Este último aspecto es el más importante de las pruebas de resistencia mecánica, porque es el que nos permiten obtener el valor de la capacidad de carga total del material y a partir de este dato, lograr informar con precisión al modelo digitalizado, vinculando a esta masa virtual a su condición real y objetiva.

Estos ensayos fueron realizados en el Laboratorio de Resistencia de Materiales de la UFPS, con la maquina universal de ensayos, de pruebas mecánicas marca EMIC DL2000. (Figura 3).

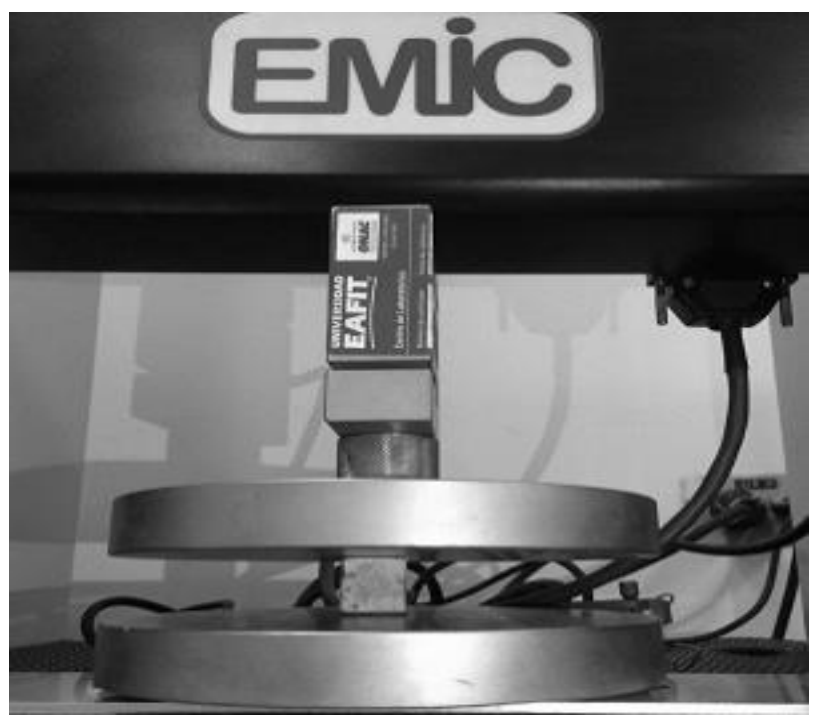

Figura 3: Proceso de pruebas de resistencia a compresión al material compuesto elaborado por estudiantes de IV semestre del Departamento de Arquitectura de la UFPS (probeta de $10 \mathrm{~cm}$ $\mathrm{x} 10 \mathrm{~cm} 10 \mathrm{~cm})$. Laboratorio de resistencia mecánica de la UFPS. Fuente: autores. 


\section{FASE 3}

Proceso Digital - Materia Informada: Según los análisis y resultados obtenidos de la maquina universal de ensayos mecánicos, se informa el modelo digital, mediante software (Scan\&Solve); esto permite simular la capacidad de carga total, máximo esfuerzo a la deformación y momento de rotura, cada vez que se encuentra sometido a las variables de trasformación que se producen en el objeto proyectual.

Estas fuerzas de cambio por decisiones de diseño inciden en la forma, influyen en el comportamiento de la materia y es desde el soporte digital y la información inicial otorgada al modelo, que se logra tener control en la toma de decisiones. Las variaciones y manipulaciones en la forma, se desarrollan a partir de software (Rhinoceros), y se controla mediante las definiciones creadas en el plugins Grasshopper, que permite el control mediante la parametrización de los cambios deseados en el material digital y la información que inciden en el objeto proyectual.

\section{RESULTADOS}

\section{PRODUCTO DIGITALIZACIÓN - MODELO}

El primer resultado, situó la forma análoga de la materia en cuestión en el mundo virtual fuera de la experiencia humana. Es decir, el objeto análogo deviene en información, a través de un proceso de escaneado 3D que resguarda los atributos iniciales de la forma de la materia en cuestión, y permite su control para sus posteriores usos, por ejemplo, modelado, fabricación, inclusión de nueva información etc. (Figura 4,5 y 6).

Así que, este proto- modelo digital u objeto virtual, a partir de este momento pasó a existir en el mundo matemático de la computadora, en la cual, los sistemas tradicionales de ideación fueron cuestionados.

En concreto, el resultado de esta fase es una hipótesis del objeto real, conformado por la captura de más de 277.000 puntos en el espacio tridimensional. Así pues, esta nube de puntos permite la manipulación de la forma, directamente en entornos virtuales.

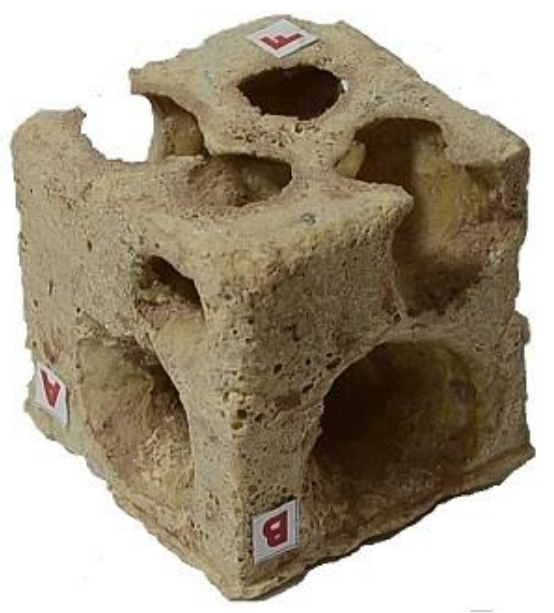

Figura 4: Probeta de material compuesto y vacíos (análoga). Fuente: autores.

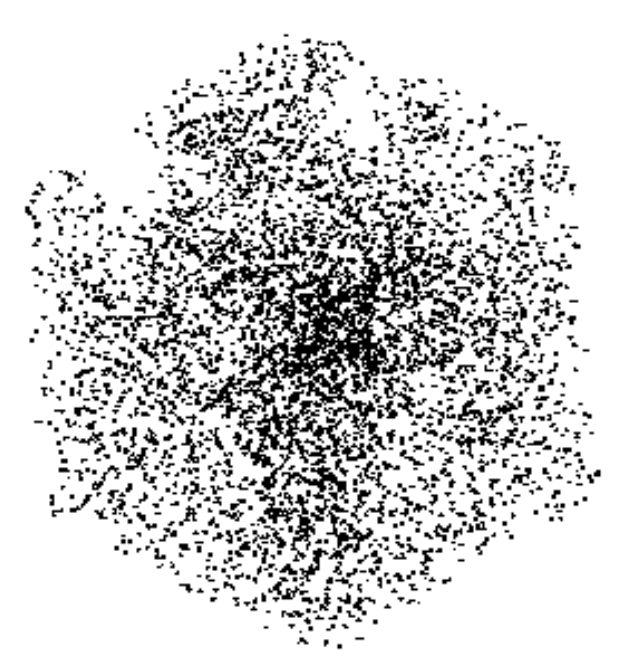

Figura 5: Nube de puntos producto escaneo digital. Fuente: autores.

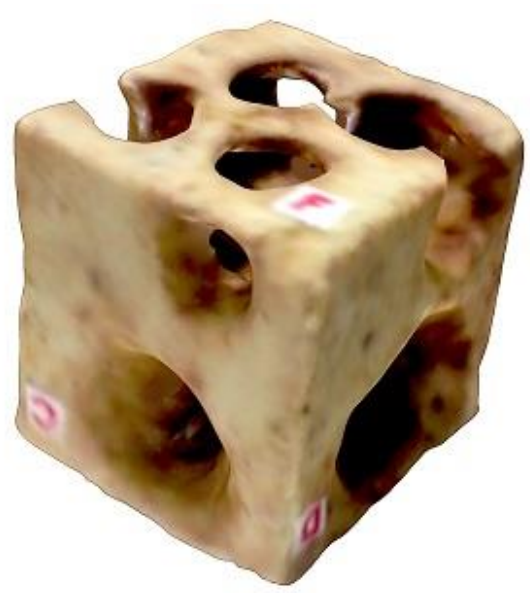

Figura 6: Simulación de la probeta de material compuesto. Fuente: autores.

\section{PRODUCTO ANÁLOGO - DATA}

De otro lado, fue necesario decodificar la materia análoga a través de su correlación con la resistencia a las dinámicas de fuerza que dan forma a su estructura. El resultado de este proceso se visibilizo a través de datos numéricos y tablas extraídos de pruebas de resistencia de materiales que desvelaron el comportamiento mecánico del material compuesto experimentado.

Dentro de los resultados que se obtuvo en el material expuesto a la prueba de ensayos mecánicos, encontramos los siguientes valores que corresponden a una probeta de un área de $370 \mathrm{~mm} 2$, resistencia máxima de 2543N, una zona límite de deformación que se encuentra en $0.5800 \mathrm{~mm} / \mathrm{mm}$, y un comportamiento elástico de 14.64Mpa. (Figura 7). 

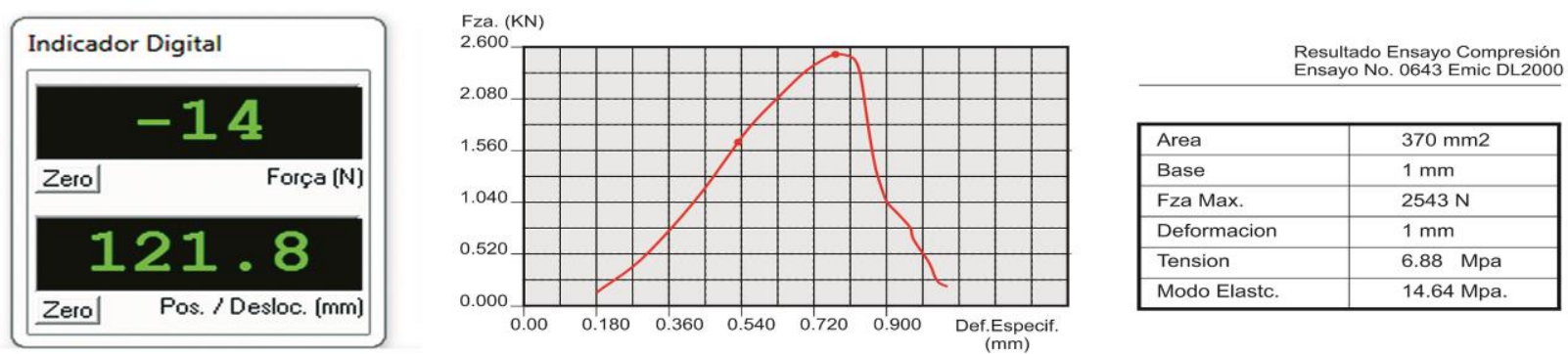

Figura 7: Grafía de extracción de información que permita informar el proto-modelo. Fuente: autores.

Tabla 1: Resultados de las pruebas de ensayos mecánicos.

\begin{tabular}{lllllll}
\hline Prob. & Area. & Base. & Fza. & Defor. & Tens. & Elas. \\
\hline UND & $\mathrm{mm} 2$ & $\mathrm{~mm}$ & $\mathrm{~N}$ & $\mathrm{~mm}$ & Mpa & Mpa \\
CP1 & 72 & 1 & 125 & 0 & 1.74 & 23.61 \\
CP2 & 370 & 1 & 2543 & 1 & 6.88 & 14.64 \\
& & & & & & \\
No.CPs & 2 & 2 & 2 & 2 & 2 & 2 \\
Media & 220. & 1.065 & 1334 & 0.374 & 4.30 & 19.1 \\
Desv. & 210. & 0.022 & 1710 & 0.387 & 3.63 & 6.33 \\
Cof.Var & 95.3 & 2.113 & 128.2 & 103.4 & 84.3 & 33.1 \\
Min & 72.0 & 1.049 & 125.2 & 0.100 & 1.73 & 14.6 \\
Max & 369. & 1.081 & 25.43 & 0.648 & 6.87 & 23.6 \\
\hline
\end{tabular}

\section{PRODUCTO DIGITAL - MATERIA INFORMADA}

Una vez, otorgadas las cualidades paramétricas de forma y resistencia al proto- modelo digital, esté fue cotejado en el espacio abstracto en relación con las dinámicas de fuerzas y la disposiciones del espacio topológico en su interior.

Esta condición abstracta significó que desapareciera de los resultados la similitud entre la forma y la representación, por ejemplo, de un dibujo en si mismo, y surgiera otros tipos de ideación y representación, a través de parámetros, relaciones y reglas, las cuales, generaron formas, algunas veces opacas para el diseñador, pero, que facilitaron su construcción, su control y la relación con otros requerimientos futuros de diseño.

Por ejemplo, los resultados de este proceso permiten establecer definiciones en Grasshopper (datos, acciones y relaciones), que desarrollaron reglas para construir, manipular y controlar el proto-modelo digital en un entorno RhinoCeros. Definiciones desarrollada por Pablo Rey

En particular, se controló el número de puntos en el espacio del objeto para facilitar las operaciones de simulación y modelado, así como también las lógicas de conexión o construcción digital del objeto a prueba, sin romper su continuidad geométrica. (Figura 8 y 9 )

Así mismo, se introdujeron componentes a las definiciones que accionaron operaciones de redistribución del espacio de su geometría topológica, que, permitieron la confrontación posterior con el software de simulación de fuerzas Scan\&Solve para Rhinoceros. (Figura 10, 11 y 12).

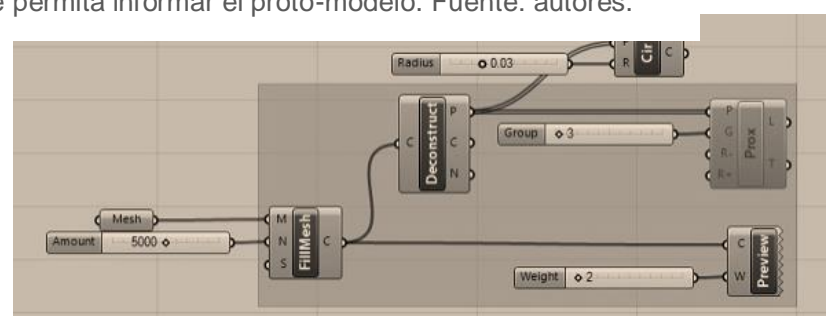

Figura 8: Definición realizada en un entorno Grasshopper de RhinoCeros, que, configura el número de puntos en el espacio, y conecta los más cercanos aleatoriamente a medida que estos puntos se desplazan en el espacio digital reinterpretando la forma original del objeto hipotético cada vez q se manipula, pero no, la geometría topológica, definicion descartada.

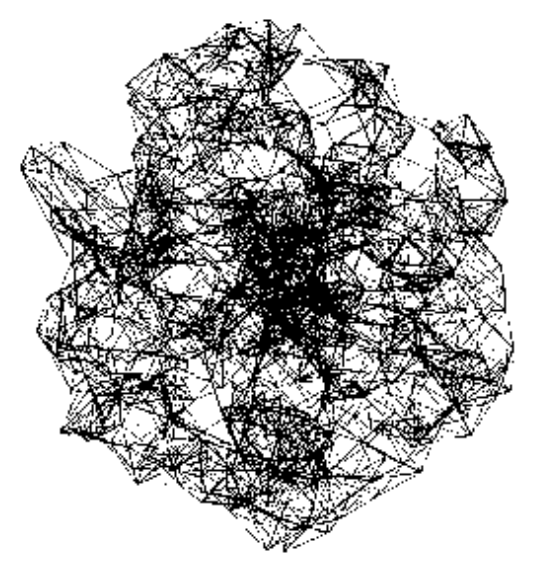

Figura 9: Visualización de la definición de la figura 8, conexión de puntos aleatorios. Fuente: autores.

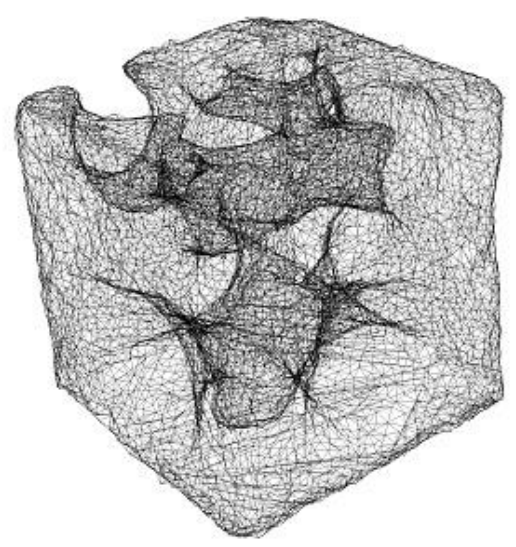

Figura 10: Visualización de partida de la definición de la figura 11 , conexión de puntos que configuran polígonos en la superficie. Fuente: autores. 


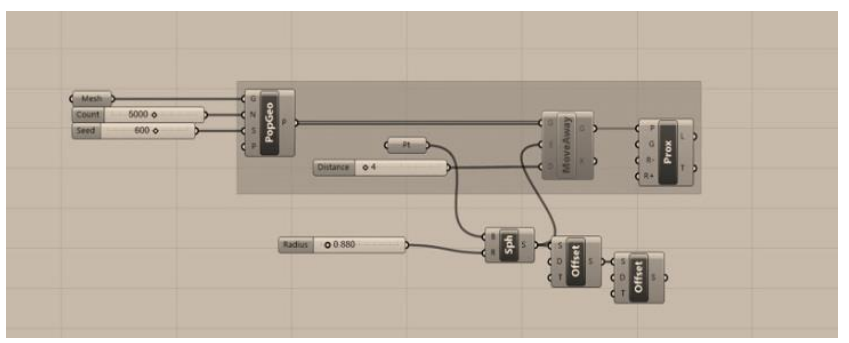

Figura 11: Definición realizada en un entorno Grasshopper de Rhinocero, que, configura una red alámbrica continua susceptible de manipularse sin perder su carácter topológico, a través de un Spin Force o fuerza de giro. Fuente: autores.

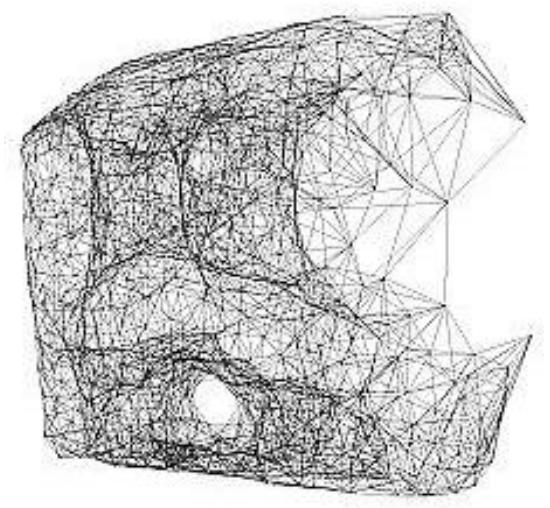

Figura 12: Visualización del alámbrico manipulado por la acción del Spin-Force de la definición de Grasshopper de la figura 11. Fuente: autores.

Por último, a partir del soporte digital Scan\&Solve de simulación estructural dentro de un entorno Rhinosceros y la información conferida al proto- modelo digital se desarrolló el análisis estructural de las correlaciones entre la materia y su resistencia en relación con las diferentes disposiciones del espacio topológico deformado, como si se visualizara la capacidad espacial bajo su suporte material, lo que facilita futuros escalonamientos y respuestas de diseños más eficientes.(Figura13).

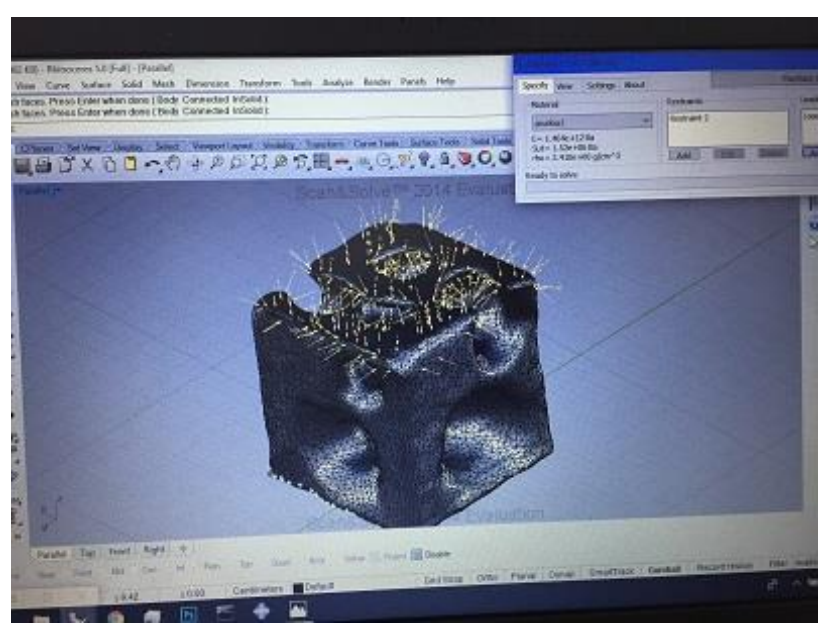

Figura 13: Visualización del entorno digital de la simulación de fuerzas, Scan\&Solve.

Este proceso de manipulacion desvela las relaciones entre la forma y la materia en cuestion, cuando, es aplicada una fuerza vertical en el eje z. Y funciona como estrategia para la creacion de espacios complejos en tiempo real. Especificamente, las experimentaciones desarrolladas confrontan el proto-modelo digital, en su estado original, para posteriormente modificar los vacios o espacios existentes en la materia.

El resultado de la primera simulacion (estado original) en un entorno Scan\&Solve la materia se desplaza y genera un riesgo de falla en la cara superior del proto-modelo del $70 \%$. En particular, esta falla se presenta en el protomodelo bajo un espacio en forma de esferoide eliptico. Como respuesta, la geometria topologica del espacio esferoidal es manipulada de forma que tienda hacia una esfera, sin perder sus atributos topologicos. Esta accion, en la segunda simulacion disminuye el desplazamiento de la materia y su falla inicial baja en $60 \%$. (Figura 14).
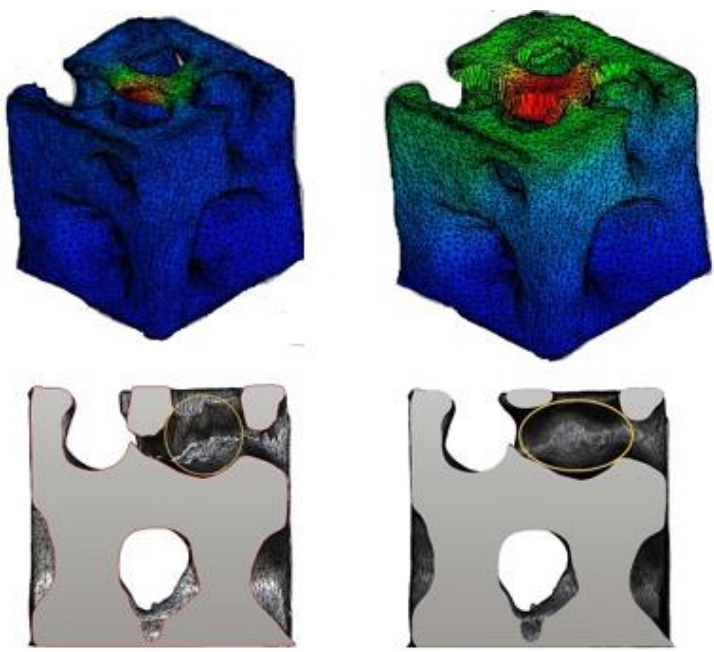

Figura 14: manipulacion del proceso de espacios o vacios dentro de la materia informada en relacion con las dinamicas de fuerzas. Fuente: autores.

Este proceso involucra al diseñador dentro de una experiencia espacio temporal simulada para la toma de decisiones más eficaces en la creación de espacios arquitectonicos en relación entre nuestra humanidad y lo real.

\section{DISCUSIÓN}

La articulación de los procesos proyectuales con un mundo objetivo, más cercano al pensamiento científico, aumenta la eficiencia en las respuestas de diseño. Porqué, ésta permite la comprensión de parámetros, relaciones y reglas de sistemas complejos, y su modelado simultaneo. Como se demuestra en el anterior ejercicio de investigación.

Por ejemplo, este ejercicio extrae la esencia del material en sí, para conferir sus condiciones a modelos abstractos con relación a la dinámica de fuerzas, que a su vez, permiten la manipulación de la materia informada, dentro de procesos de diseño.

Así pues, este ejercicio le confiere voz a la materia, para que ésta explique, que quiere ser. Es decir, la materia informada podrá responder a la cuestión sobre el querer ser del material, planteada por Louis I. Kahn a mitad del siglo $\mathrm{XX}$, ya no, como una pregunta retórica o una 
metáfora, si no, como un soporte para las decisiones del diseñador. (Kahn, 1986)

Por ejemplo, de esta ponencia resulta el control, o por lo menos la comprensión espacio temporal de la cualidades matericas de resistencia del modelo informado, la geometría de sus vacíos, o de su espacio continuo y conectivo (topológico), en relación con la dinámicas de fuerzas y el objeto proyectual. Algo impensable si operamos en el mundo estático del dibujo y de las percepciones sensoriales humanas.

En otras palabras, esta ponencia cuestiona el divorcio entre el diseño y su construcción acaecido en la disciplina de la arquitectura, desde ya hace más de 500 años en tiempos de Leon Bauttista Albertí y su tratado "De Re Aedificatoria". Es decir, cuando, el dibujo reemplazo la forma de hacer del arquitecto en tiempo real de los maestros del Gótico. (Ross, 2014)

Por tanto, en sincronía con Ross, Baudrillard, esta ponencia debate el uso único de las representaciones tradicionales del dibujo y propendemos por la simulación como medio alternativo de representación para resolver problemas de diseño y fabricación. A pesar de las elementales limitaciones de la simulación, por operar en un plano hipotético.

Es decir, por ejemplo, esta ponencia que actúa dentro de esta especie de universo matemático, planteado por Slavoj Zizeck. La cual, opera dentro de software de simulación, o tecnologías de captura de datos, que en principio están predeterminadas por su programador y/o creador, y no por el diseñador que actúa como su operario, y que muchas veces esta distanciado de las lógicas que gobiernan estas tecnologías.

No obstante, las operaciones de diseño en este universo, al final, sobrepasan los alcances de respuesta de las operaciones del diseño tradicional, y de la humanidad del diseñador. Por tanto, los procesos de ideación dentro de estos límites tradicionales disciplinares del diseño, Indefectiblemente tendrá que variar.

$Y$, en lugar de responder con complicados y laboriosos dibujos, la tarea será comprender los parámetros, relaciones y reglas de esa "realidad objetiva" y responder creando diversas técnicas que den respuestas de diseño más eficientes, a nuevas problemáticas más complejas, las cuales, surgen conforme los humanos aumentamos nuestro conocimiento.

Este proceso de manipulacion con miras a estableser una estrategia de ideacion en el diseño arquitectonico, es el inicio de futuras experimentaciones en el marco del proyecto de investigacion: Experimentacion con materiales y sus formas, de la cual, es parte esta ponencia. Estas tareas ocurren en el seno del laboratorio de diseño de la UFPS (grupo de investigacion en diseño, ciencia y tecnologia-dlab)

\section{AGRADECIMENTOS}

Agradecemos a:

- Universidad Francisco de Paula Santander, UFPS Colombia, especialmente a su comité administrativo por financiar las pruebas de resistencia mecánica del material estudio.

- Laboratorios de resistencia mecánica de Ingeniería, y simulación del departamento de arquitectura, SIMU-Lab de la UFPS, por sus servicios de prueba de resistencia, escaneo 3D y software de simulación respectivamente.

- Docentes $\mathrm{PhD}$ en materiales avanzados, licenciado en física, Gabriel Peña, March José Miguel Jaimes, March Pablo Serrano y March Javier Lemus por su acompañamiento y colaboración en el proceso de creación de los materiales compuestos.

- Beca especial del SIMU-Lab y de arquitectura de VI semestre Pablo Rey por la operación de los software de simulación y modelado empleados. Así mismo, agradecemos al estudiante Alejandro Silva C por el proceso de escaneo digital 3D del material compuesto análogo.

- Estudiantes de arquitectura de taller de diseño IV semestre I. II, 2017, del Departamento de Arquitectura por su trabajo en la creación de materiales compuestos.

\section{REFERENCIAS}

Baudrillard, J. (1978). Cultura y Simulacro. Barcelona: Editorial Kairos.

Fuller, R. B. (1975). Synergetic Exploration in the Geometry of Thinking. New York: Macmillan Publishing.

Le Ricolais, R., "Things themselves are lying, so are their images." Inter- views with Robert Le Ricolais, en Vía 2,. 1973, p. 88.

Juárez, A. (1996). El arte de construir con agujeros, reflexiones en torno a Robert Le Ricolaris. (L. Mansilla, L. Rojo, \& E. Tuñon, Eds.) Circo , 39, 1-11.

Ross, D. S. (2014). The Death of Drawing: Architecture in the Age of Simulation. London \& New York: Routledge.

Thompson, D. W. (1942). On Growth and Form (2 ed.). Cambridge and New York: University Press and Macmillan.

Kahn, L. I. (1986). The Invisible City-International Design Conferens at Aspen Colorado, 19 June 1972. In R. S. Wurman, What Will Be Has Always Been The Word of Louis Kahn. New York: ACCESSPRESS Ltda. P. 152

Zizek, S. (2008). (S. I.-S. streets", Producer) From https://www.youtube.com:https://www.youtube.com/watch?v=WX 8Yju8jeA4

ŽIŽEK, S. (2014). ABSOLUTE RECOIL TOWARDS A NEW FOUNDATION OF DIALECTICAL MATERIALISM . Verso. 